Ingeniería, Industria y Construcción

El capital humano y la productividad de las empresas

\title{
Human capital and the productivity of companies
}

\author{
Díaz Díaz, América Alejandra; Toscano Moctezuma, Juan Alfonso
}

\author{
América Alejandra Díaz Díaz \\ america.adiaz@outlook.com \\ Universidad Autónoma de Ciudad Juárez, México \\ Juan Alfonso Toscano Moctezuma \\ jtoscano@uacj.mx \\ Universidad Autónoma de Ciudad Juárez, México
}

Revista Torreón Universitario

Universidad Nacional Autónoma de Nicaragua-Managua,

Nicaragua

ISSN: 2410-5708

ISSN-e: 2313-7215

Periodicidad: Cuatrimestral

vol. 11 , núm. 30, 2022

revis.torreon.faremc@unan.edu.ni

Recepción: 17 Noviembre 2021

Aprobación: 09 Diciembre 2021

URL: http://portal.amelica.org/ameli/

DOI: https://doi.org/10.5377/rtu.v11i30.13427

El autor o los autores de los artículos, ensayos o investigaciones conceden a la Universidad Nacional Autónoma de Nicaragua, Managua (UNAN-Managua) los derechos de edición (copyright) del trabajo enviado, por consiguiente la Universidad cuenta con el derecho exclusivo para publicar el artículo durante el periodo completo de los derechos de autor.

\section{(c) (1) 9}

Esta obra está bajo una Licencia Creative Commons AtribuciónNoComercial-SinDerivar 4.0 Internacional.
Resumen: El presente artículo pretende presentar los factores relacionados con el capital humano y su impacto en la productividad de las empresas, esto a través de una revisión literaria. El objetivo del artículo es describir la influencia del capital humano en el cumplimiento de los objetivos de productividad en las organizaciones. La metodología se basó en la búsqueda de artículos con criterios de inclusión y exclusión específicos de los cuales se extrajeron 30 artículos y se seleccionaron 14 para revisión.

Palabras clave: capital humano, desempeño, gestión del capital humano, productividad.

Abstract: This article aims to present the factors related to human capital and its impact on the productivity of companies, this through a literary review. The objective of the article is to be able to describe the importance and influence of human capital in compliance of productivity objectives in organizations. The methodology was based on the search for articles with specific inclusion and exclusion criteria, from which 30 articles were extracted and 14 were selected for review.

Keywords: Human capital, performance, human capital management, productivity.

\section{INTRODUCCIÓN}

Se considera que las empresas se desarrollarán en base a los estímulos del entorno, aquellas empresas que no se desarrollan dejan de existir. De acuerdo con (Armas et al., 2017) las empresas no son estructuras físicas si no pueden considerarse entes vivos, ya que su operación depende de las personas que la conforman, así como el cumplimiento de los objetivos o los fracasos que lleguen a presentar son en gran medida atribuidos a las personas, ya que es bien sabido que forman parte de la dirección operativa y administrativa de la organización (Armas et al., 2017, p. 5).

Se considera de vital importancia dar un vistazo hacia atrás para recapitular sobre la evolución que han tenido las organizaciones a través del tiempo, así como los principios teóricos que han aportado al desarrollo 
de la gestión del talento humano, la gestión del talento humano va más allá de los talentos que pueda poseer una persona (Armas et al., 2017).

En la ilustración 1 se incluyen los aspectos fundamentales que (Armas et al., 2017) enlistan y que se suscitan en las diferentes eras del desarrollo en las organizaciones: Era Industrial Clásica; Era Neoclásica y la Era de la Gestión del Conocimiento. En estas diferentes eras se puede apreciar, por un lado, la evolución de las organizaciones y principios de la administración y por el otro, las características de las relaciones laborales en su evolución.

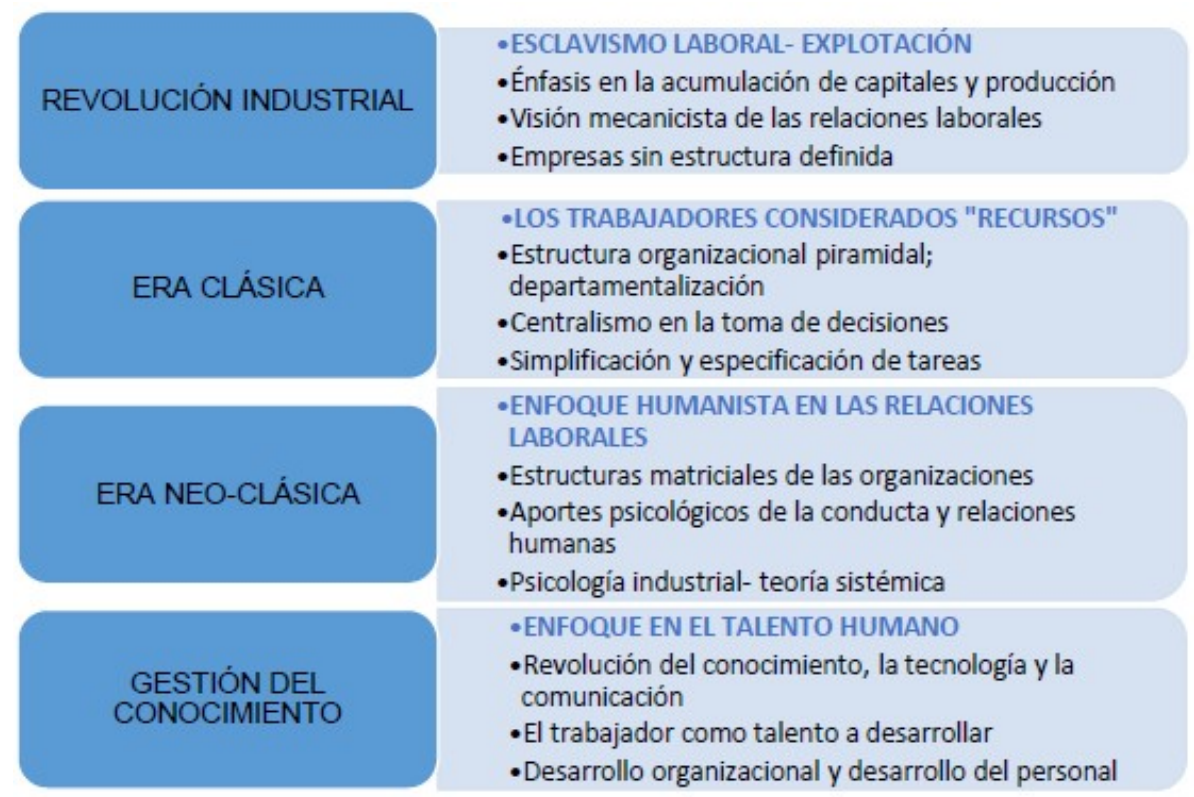

ILUSTRACIÓN 1.

Síntesis de los cambios globales en las organizaciones y las relaciones laborales. Fuente: (Armas et al., 2017).

En la ilustración 1, se presenta un resumen de las diferentes eras en el desarrollo del capital humano dentro de una organización, en cada era se puede apreciar el su avance y en la última etapa resalta el talento humano como un punto clave para las empresas, que es uno de los temas a tratar en este artículo.

De los principales factores que intervienen en el desarrollo de una empresa es la productividad, (Hinojo et al., 2020) "la definen como el alcance de las metas utilizando los mínimos recursos para producir en menor o igual cantidad de tiempo que usando una gran variedad de ellos" (p. 52).

"La productividad es el resultado de la articulación armónica entre la tecnología, la organización y el talento humano, combinando en forma óptima o equilibrada los recursos para la obtención de los objetivos" (Jaimes et al., 2018).

\section{Desarrollo}

\subsection{Metodología para la revisión de la literatura.}

Los trabajos incluidos en la revisión de la literatura abarcaron aquellos que relacionaban el desempeño del capital humano y la productividad. Se utilizó el buscador Google Académico para seleccionar los artículos relacionados con el tema.

En total, en forma automática se visualizaron 98,600 documentos, dentro de los cuales se encontraron artículos del sector industrial, instituciones educativas y microempresas. 
Estas publicaciones en su mayoría, trataban de aspectos amplios del tema de interés, por lo que se establecieron los siguientes criterios de selección:

Criterios de inclusión.

- CI 1. Investigaciones con fecha de publicación del 2015 a la fecha.

- CI 2. Investigaciones donde se pudiera identificar de forma rápida en el en el resumen o palabras clave "talento humano", "capital humano", "desempeño del capital humano" en relación con "la productividad".

- CI 3. Investigaciones donde dentro del propósito u objetivo se incluía el estudio de la relación, influencia o impacto del desempeño del capital humano en la productividad.

- CI 4. Se estableció con base al funcionamiento de la búsqueda, es decir, se para la búsqueda se establecieron palabras clave como: factor humano, productividad, motivación, competencias, desempeño laboral, talento humano.

- CI 5. Análisis de revistas existentes en Google Académico y plataformas científicas como https://w ww.redalyc.org/, http://www.scielo.org.pe/, etc., y libros también existentes en Google Académico.

Criterios de exclusión.

- CE 1. Investigaciones publicadas del 2014 y años anteriores.

- CE 2. Estudios realizados en instituciones de salud y educación.

En total se revisó el detalle de 30 documentos de los cuales fueron seleccionados 14, de estos mismos se extrajo la información relevante de acuerdo al objetivo de esta investigación y a los criterios antes mencionados.

A continuación, se presenta una descripción general de los documentos considerados en la investigación: 


\begin{tabular}{cccc}
\hline No. & Revista & País & Motivo de selección \\
\hline 1 & Innovar & España & CI 1, CI 2, CI, 4 \\
2 & Eca Sinergia & Ecuador & CI 1, CI 2, CI 4 \\
3 & Innovar & Colombia & CI 1, CI 2, CI 4 \\
4 & Información Tecnológica & Colombia & CI 1, CI 2, CI 3, CI 4 \\
5 & Didáctica y Educación & Ecuador & CI 1, CI 2, CI 4 \\
6 & Universidad privada del norte & Perú & CI 1, CI 2, CI 3, CI 4 \\
7 & Revista Venezolana de Gerencia & Venezuela & CI 1, CI 2, CI 4 \\
8 & Teckne & Colombia & CI 1, CI 2, CI 3, CI 4 \\
9 & Depto. de Administración, & Ecuador & CI 1, CI 2, CI 3, CI 4 \\
& Economía y Finanzas & Colombia & CI 1, CI 2, CI 3, CI 4 \\
10 & Innovar & Perú & CI 1, CI 2, CI 4 \\
11 & Ciencia y tecnología & - & CI 1, CI 2, CI 4 \\
12 & Investigación y negocios & Ecuador & CI 1, CI 2, CI 4 \\
13 & Gestión Joven & Argentina & CI 1, CI 2, CI 4 \\
14 & Visión de futuro & & \\
\hline
\end{tabular}

\subsection{Revisión de la literatura.}

\subsubsection{El capital humano como elemento estratégico.}

El principal objetivo de una empresa es el de posicionarse frente a su competencia como una empresa innovadora y ofreciendo calidad en servicios o productos superior al resto, esto como parte de sus estrategias en ventajas competitivas, para esto se apoyan en las capacidades y habilidad del talento humano como una de tus principales fortalezas para el desarrollo de dichos objetivos (Montoya et al., 2016).

Por lo anterior podemos decir que, si el personal desarrolla un nivel de conocimiento superior, así como competencias y habilidades esto facilita su colaboración dentro de la empresa y la realización de sus tareas y actividad asignadas de manera eficiente y la calidad superior al estándar y con posibilidad de agregar valor agregado a la organización.

Cuando una organización incluye dentro de su plantilla a personal con las características anteriores, dicha organización presenta mejoras en la productividad y se posiciona como una empresa competitiva que se traduce a productos o servicios que destacan del resto de las organizaciones (Simancas et al., 2018).

Se puede considerar también al talento humano como capital que entre más acumula conocimientos, habilidades y experiencia que obtienen a través de la educación y experiencia aprendida en la empresa aporta valor agregado a la misma. 
Se considera que se está invirtiendo en capital humano, cuando se invierte en la capacitación en la operación, en los servicios de salud que se les ofrece, las recompensas y prestaciones, en resumen, todo el posible ingreso que pueda tener el personal como beneficio por parte de la organización. (Simancas et al., 2018).

Los especialistas en gestión estratégica de recursos humanos teorizan que, a través de sus prácticas de recursos humanos, como el desarrollo de personal o capacitación, las organizaciones crean una forma particular de conocimiento agregado, habilidades y competencias que a su vez contribuye al desempeño a nivel organizacional (Simancas et al., 2018).

\subsubsection{Gestión y desarrollo del capital humano.}

La planeación, organización, el desarrollo, la coordinación y el control son capaces en combinación de crear el ambiente ideal para el desempeño eficiente del personal, esto se puede generar si la organización construye un entorno que permita que las personas que colaboran en ella alcancen sus objetivos individuales, aunque estos no estén directamente relacionados con la organización, sin embargo, al alcanzar sus objetivos personales se verá reflejado de formato colectiva dentro de la empresa (Ramírez y Zavaleta, 2017).

El objetivo de la gestión del capital humano incluye el desarrollo de procedimiento que afiancen y definan las estructuras de la empresa de manera eficiente, así como el firme establecimiento de mecanismos que contribuyan al logro de los objetivos de la empresa, para lograr lo anterior, se debe disponer de técnicas dirigidas hacia la planeación, la organización, la dirección y el control como cualquier proceso administrativo, pero con enfoque en el personal (Vera y Blanco 2019).

'El objetivo general de la gestión del talento humano es la correcta integración de la estrategia, la estructura, los sistemas de trabajo y las personas, con la finalidad de lograr de las personas el despliegue de todas sus habilidades y capacidades y lograr la eficiencia y la competitividad organizacional" (Vera y Blanco 2019). Lo anterior se puede traducir en alcanzar un nivel máximo en productividad dirigiendo los esfuerzos en propiciar un clima laboral ideal para que el personal se pueda desenvolver de tal manera que se logre el objetivo de productividad previsto (Maturana y Andrade, 2019).

De este objetivo general se desprenden varios objetivos específicos tales como (Vera y Blanco 2019).

1. Ayudar a la organización a alcanzar sus objetivos.

2. Proveer a la organización empleados bien entrenados y motivados.

3. Desarrollar y mantener la calidad del ambiente de trabajo.

4. Proporcionar competitividad a la organización.

5. Permitir la autorrealización y la satisfacción de los empleados en el trabajo.

6. Administrar el cambio e instruir herramienta para lograrlo.

También se puede nombrar los objetivos generales que servirán para alcanzar la eficiencia en la organización como (Vera y Blanco 2019).

1. Atraer: Es la manera de buscar y atraer a las personas más capaces a la empresa. Son las estrategias y tácticas para que las personas tengan mucho interés en trabajar en nuestra organización.

2. Desarrollar: Para potencializar la capacidad dentro de esta fase se puede focalizar en los siguientes factores:

- El entrenamiento, que es propiciar el ambiente para que el personal adquiera habilidades que le permitan realizar su trabajo con productividad.

- La capacitación, es el aprender cómo hacer el trabajo. 
- Desarrollo del personal, referido a identificar motivaciones y deseos que impulsan a desempeñar un trabajo con entusiasmo.

- Desarrollo organizacional: Enseñar a pensar acorde a la organización como los valores, metas, comportamiento y proyecciones.

3. Retener: Son las políticas, estrategias y tácticas que se utiliza para que las personas que trabajan con la empresa puedan quedarse por muchos años y en esta parte entra algo muy importante que es la motivación.

\subsubsection{La motivación y el capital humano.}

"La motivación es el proceso de estimular a un individuo para que se realice una acción que satisfaga alguna de sus necesidades y alcance alguna meta deseada para el motivador" (Miranda, 2016).

"La motivación de los recursos humanos consiste fundamentalmente en mantener culturas y valores corporativos que conduzcan a un alto desempeño" (Miranda, 2016).

La motivación es el deseo de hacer mucho esfuerzo por alcanzar las metas de la organización, condicionado por la necesidad de satisfacer alguna necesidad individual (Miranda, 2016).

Por lo anterior, la motivación se puede definir como los medios que el personal y la empresa propician para lograr el cumplimiento de sus metas con el fin de verse reflejado el interés personal y colectivo.

La motivación es la principal fuente de retención y satisfacción del personal de una organización, ya que, aunque el personal sea parte de una organización no separan sus metas y sueños personal por este simple hecho, la empresa debe motivarlas para que cumplan dichas metas y sueños lo cual será recompensado con algún factor de interés de la persona o grupo de personas hacia la organización (Miranda, 2016).

\subsubsection{El capital humano y la productividad.}

La productividad es el principal objetivo de una organización, los esfuerzos del capital humanos están enfocados con el cumplimiento de este que es el objetivo principal (Suarez, 2018).

Los recursos de la organización son administrados por personas quienes enfocan su actividad y esfuerzos en producir productos o servicios de calidad superior con respecto a la competencia y logrando la mejora de sus actividades día con día para obtener un impacto positivo dentro de la organización, así como en su vida personal (Obando, 2020).

El desarrollo de las habilidades del personal enfocado a la mejora de la productividad se puede obtener realizando una inversión en la capacitación e incrementar la experiencia de las personas dentro de la misma empresa (Maturana y Andrade, 2019). En general dentro de las organizaciones se puede identificar que los empleados aumentan la productividad cuando aprenden nuevas habilidades, cuando exploran nuevos métodos para realizar sus actividades, al perfeccionar las técnicas ya aprendidas en instituciones educativas o empresas a las cuales pertenecieron con anterioridad (Ganga y Villacís, 2018).

Partiendo de lo anterior el talento adquirido por los empleados y acumulado a través de la educación o experiencia, así como las propias habilidades natas de la persona sirven para propiciar y potenciar la mejora y apoyado en actividades de capacitación puede resultar en la explotación de talento encaminado al bien de la organización (Simancas et al., 2018).

(Simancas et al., 2018) mencionan que se debe enfocar los esfuerzos en tres tipos de acciones relacionadas con el individuo para incrementar la productividad:

- Motivación, competencias, satisfacción laboral, identificación, compromiso y empoderamiento con la organización. 
- Aspectos generales como la participación, la cohesión y la gestión de conflictos.

- Fortalecer la cooperación haciendo énfasis en la cultura organizacional, clima y liderazgo.

Para determinar si las estrategias implementadas relacionadas con la mejora en la productividad y su impacto en la misma, es común la implementación de evaluaciones esto se logra definiendo indicadores para poder medir la productividad dentro de la organización (Diaz y Quintana, 2021). Los principales indicadores utilizados comúnmente en las empresas son la eficiencia, la eficacia y la efectividad, estos factores al escucharlos son relacionados inmediatamente con la productividad. Los factores anteriores permiten medir el nivel de productividad alcanzado y adicional el impacto o la influencia del personal en ese resultado, con ello se puede analizar si el nivel de productividad alcanzado fue el previsto o supero lo definido por la empresa (Simancas et al., 2018).

La productividad y el desempeño laboral del personal dentro de una empresa son factores de gran relevancia para el cumplimiento de los objetivos de la organización, el enfoque principal de estos factores se centra en las competencias y la contribución de estas y de la mano de las habilidades del trabajador orientadas hacia el eficiente desempeño (Hinojo et al., 2020).

Las competencias se definen como "el conjunto de características de una persona que están relacionadas directamente con una buena ejecución en una determinada tarea o puesto de trabajo". Por lo anterior podemos decir se puede generar un empleado competente capacitándolo para incrementar el rendimiento en una tarea específica; "por ejemplo, una persona competente en resolución de problemas dará respuesta a las posibles dificultades de un modo más eficaz y rápido que otra persona con baja competencia resolutiva" (Hinojo et al., 2020).

La evaluación de desempeño laboral es un hecho cotidiano de la vida, así como en la organización motivada por la experiencia laboral y es una herramienta fundamental para el desarrollo del talento humano en una organización (Álvarez et al., 2018).

\section{CONCLUSIONES}

En el entorno de las organizaciones un factor importante para la productividad son los costos debido a que, para alcanzar la productividad, la empresa tiene que administrar correctamente los recursos que esto conlleva, de tal manera que se alcance el objetivo de productividad sin poner en riesgo el buen desempeño financiero de la organización.

Para lograrlo, el capital humano de la empresa necesariamente debe estar comprometida, capacitada y motivada, para que trabaje en dirección del objetivo de productividad establecido por la empresa.

La productividad de una empresa es el reflejo de la capacitación del personal de la misma, por lo anterior cada vez es más común que las empresas consideren invertir en la capacitación de sus trabajadores para obtener resultados positivos en el futuro.

Ahora bien, la literatura seleccionada para el tema evidencia la creciente importancia del capital humano para la productividad de las organizaciones, donde queda a relucir que el conocimiento, las competencias y las habilidades que las organizaciones implantan en sus trabajadores son consideradas ventajas competitivas en el mercado.

Por lo anterior se puede obtener como resultado principal de esta revisión de literatura que el desempeño del talento humano es pieza clave para alcanzar el objetivo de productividad en una organización.

Para que el desempeño del personal en una organización logre posicionarse en un nivel con el cual se pueda alcanzar la productividad objetivo, las empresas deben concentrar sus esfuerzos en la capacitación del personal

Durante las diferentes etapas del desarrollo de las organizaciones era normal considerar al trabajador como un recurso más de producción a explotar en las empresas, sin embargo, actualmente se valora y resalta el rol del personal para la mejora de la productividad. 
En una organización el capital humano es el recurso de más importancia, la cobertura de sus necesidades dentro de la organización, el aumento de su potencial y el desarrollo de cada trabajador es de alta influencia para el cumplimiento de los objetivos de la empresa, entre ellos la productividad.

\section{REFERENCIAS}

Álvarez Indacochea, Blanca; Alfonso Porraspita, Deysi; Indacochea Ganchozo, Blanca. (2018). El desempeño laboral: un problema social de la ciencia. Didáctica y Educación, NA, 1-12.

Armas Ortega, Yadira; Llanos Encalda, Mónica del Pilar; Traverso Holguín, Paola. (2017). Gestión del talento humano y nuevos escenarios laborales. Samborondón - Ecuador: Universidad ECOTEC.

Diaz Muñoz, Gabriel Alejandro; Quintana Lombeida, María Dolores. (2021). La gestión del talento humano y su influencia en la productividad de la organización. Gestión Joven, 22, 29-48.

Ganga Contreras, Francisco; Villacís Moyano, Horacio. (2018). Factores individuales y grupales que influyen en la productividad de los trabajadores de las grandes y medianas empresas de Guayaquil. Perspectivas, 42, 97-122.

Hinojo Lucena, Francisco Javier; Aznar Díaz, Inmaculada; Romero Rodríguez, José María. (2020). Factor humano en la productividad empresarial: un enfoque desde el análisis de las competencias transversales. INNOVAR, 30, $52-63$.

Jaimes, Ludym; Luzardo, Marianela; Rojas, Miguel. (2018). Factores determinantes de la productividad laborar en pequeñas y medianas empresas de confecciones del área metropolitana de Bucaramanga, Colombia. Información Tecnológica, 29, 175-186.

Maturana, Diana; Andrade, Verónica. (2019). La relación entre la formalización de las prácticas de gestión humana y la productividad de las mipymes. INNOVAR, 29, 101-114.

Miranda Hayes, Danahe. (2016). Motivación del talento humano: La clave del éxito de una empresa. Revista digital investigación y negocios, NA, 20-27.

Montoya Agudelo, César Alveiro; Boyero Saavedra, Martín Ramiro. (2016). El recurso humano como elemento fundamental para la gestión de calidad y la competitividad organizacional. Visión de Futuro, 20, 1-20.

Obando Changuán, Marcelo Patricio. (2020). Capacitación del talento humano y productividad: Una revisión literaria. ECA Sinergia, 11, 166-173.

Ramírez García, Jaime; Zavaleta León, Víctor. (2017). Inteligencia Emocional y Desempeño Laboral en una Empresa Constructora Privada. Ciencia y tecnología, 14, 67-79.

Simancas Trujillo, Ricardo Antonio; Silvera Sarmiento, Astelio de Jesús; Garcés Giraldo, Luis Fernando. (2018). Administración de recursos humanos: factor estratégico de productividad empresarial en pymes de Barranquilla. Revista Venezolana de Gerencia, 23, 377-388.

Suarez Castro, R. M. (2018). Estrategias administrativas para la mejora de la productividad laboral y su impacto en el factor humano. TECKNE, 16, 10-13.

Vera Barbosa, Adriana; Blanco Ariza, Ana. (2019). Modelo para la gestión del talento humano en las pymes del sector de servicios de barranquilla, Colombia. INNOVAR, 29, 25-44. 